\title{
GRIM-19-mediated Stat3 activation is a determinant for resveratrol-induced proliferation and cytotoxicity in cervical tumor-derived cell lines
}

\author{
YONG-GUANG LI* , HONG-JUAN XIA* , JIAN-PING TAO, PING XIN, \\ MING-YA LIU, JING-BO LI, WEI ZHU and MENG WEI \\ Division of Cardiology, Sixth Hospital, Shanghai Jiao Tong University, \\ State Key Discipline Division, Shanghai 200233, P.R. China \\ Received September 15, 2013; Accepted July 21, 2014
}

DOI: $10.3892 / \mathrm{mmr} .2014 .2797$

\begin{abstract}
Resveratrol is a natural phenol, produced from red grapes, berries and peanuts. Previous studies have suggested that resveratrol exerts anticancer effects. Activation of the signal transducer and activator of transcription 3 (Stat3) is important in cancer. However, the mechanisms by which resveratrol suppresses the Stat 3 signaling pathway remain to be elucidated. The aim of the present study was to investigate the effects of resveratrol on GRIM-19-Stat3 signaling in HeLa cells, derived from a cervical tumor. HeLa cells were divided into experimental groups and treated with resveratrol. Western blotting was used to analyze the expression levels of p-Stat3, Stat3, GRIM-19 and $\beta$-actin. Cell viability was determined using an MTT assay. The results showed that $100 \mu \mathrm{M}$ resveratrol suppressed the proliferation and Stat 3 phosphorylation in HeLa cells, and induced the expression of the gene associated with retinoid-IFN-induced mortality 19 (GRIM-19) protein. Overexpression of GRIM-19 suppressed the Stat3 signaling pathway in HeLa cells. The Stat 3 signaling pathway was activated following the downregulation of GRIM-19 expression using short interfering RNAs (siRNAs). Resveratrol suppressed cell proliferation, however, this effect was decreased through
\end{abstract}

Correspondence to: Professor Meng Wei, Division of Cardiology, Sixth Hospital, Shanghai Jiao Tong University, State Key Discipline Division, 600 Yishan Road, Shanghai 200233, P.R. China

E-mail: weimeng_sjtu6h@163.com

*Contributed equally

Abbreviations: DMSO, dimethyl sulfoxide; DMEM, Dulbecco's modified Eagle's medium; GRIM-19, gene associated with retinoidIFN-induced mortality 19; MTT, 3-(4,5-dimethylthiazol-2-yl)2,5-diphenyltetrazolium bromide; Stat3, signal transducer and activator of transcription 3; FBS, fetal bovine serum

Key words: resveratrol, proliferation, cytotoxicity, signal transducer and activator of transcription 3 , gene associated with retinoid-IFN-induced mortality 19 , HeLa cells the use of siRNAs. The suppression of Stat 3 phosphorylation by resveratrol decreased following treatment with siRNAs. To the best of our knowledge, the present study is among the first to identify GRIM-19-Stat3 signaling as a target of resveratrol, and further elucidates the mechanisms underlying the antitumor activity of resveratrol.

\section{Introduction}

Resveratrol is a naturally occurring phytoalexin, abundant in red grapes, which possesses antioxidant and anti-inflammatory properties. Previous studies have demonstrated that resveratrol in association with the consumption of red wine exhibits cardioprotective effects (1). In addition, several studies have demonstrated that resveratrol is important in the prevention of skin cancer (2), human breast cancer (3), oral squamous cell carcinoma (4) and the inhibition of angiogenesis (5), thereby suggesting that resveratrol has anticancer properties. However, the molecular mechanisms underlying the biological effects conferred by resveratrol have not been fully defined.

The signal transducer and activator of transcription (STAT) family is important in cells and is able to promote cell proliferation and other biological processes, which can be triggered by cytokines or growth factors $(6,7)$. STAT is activated by phosphorylation of a critical tyrosine residue, which then forms dimers between two phosphorylated STAT monomers. Following this, the dimers are translocated into the nucleus where STAT regulates the expression of its target genes. Within the STAT family, Stat3 is constitutively activated in diverse types of human tumor. Constitutively active Stat 3 is able to induce oncogenic processes, growth, survival and angiogenesis (8-10), while the suppression of phosphorylated Stat3 (p-Stat3) induces the suppression of tumor growth and apoptosis $(11,12)$. Previous studies have demonstrated that p-Stat 3 is able to be downregulated by gene associated with retinoid-IFN-induced mortality 19 (GRIM-19) $(13,14)$.

Several studies have reported that the GRIM-19 protein can interact with the Stat3 signaling pathway. GRIM-19 has become a novel anticancer target in cancer cells that have constitutively active Stat3 $(15,16)$. Given that GRIM-19 and Stat 3 are present and active in many types of human 
tumor (17-19), there is considerable potential for resveratrol to modulate signal transduction pathways involved in tumor progression. The present study investigated $t$ (he effects of resveratrol on GRIM-19-Stat3 signaling in HeLa cells, which were derived from a cervical tumor.

\section{Materials and methods}

Cell lines, reagents and treatment conditions. The HeLa cell line was obtained from the American Type Culture Collection (Manassas, VA, USA). Resveratrol and 3-(4,5-dimethylthiazol-2-yl)-2,5-diphenyltetrazolium bromide (MTT) were purchased from Sigma (St. Louis, MO, USA). Antibodies against Stat3 and p-Stat3 (phosphorylated at tyrosine 705) were purchased from Cell Signaling Technology (Beverly, MA, USA). Antibodies against $\beta$-actin were purchased from Santa Cruz Biotechnology, Inc. (Santa Cruz, CA, USA). Antibodies against GRIM-19 were purchased from eBioscience (San Diego, CA, USA). Secondary antibodies were purchased from Beijing Biosynthesis Biotechnology Co., Ltd. (Beijing, China). Penicillin, streptomycin, Dulbecco's modified Eagle's medium (DMEM) and fetal bovine serum (FBS) were obtained from Gibco-BRL (Grand Island, NY, USA). HeLa cells were grown in DMEM supplemented with $10 \%$ heat-inactivated FBS, $100 \mathrm{U} / \mathrm{ml}$ penicillin and $100 \mathrm{mg} / \mathrm{ml}$ streptomycin for $48 \mathrm{~h}$ at $37^{\circ} \mathrm{C} / 5 \% \mathrm{CO}_{2}$. The HeLa cells were divided into experimental groups and each treatment condition was a single dose of resveratrol at the indicated concentration. The vehicle control was $0.1 \%$ dimethyl sulfoxide (DMSO).

Western blot analysis. Western blotting was used to analyze the expression levels of p-Stat3, Stat3, GRIM-19 and $\beta$-actin. The cells were lysed with RIPA buffer (50 mM Tris- $\mathrm{HCl} \mathrm{pH} 7.4$, $150 \mathrm{mM} \mathrm{NaCl}, 1 \%$ sodium deoxycholate, $1 \% \mathrm{NP}-40,1 \mathrm{mM}$ phenylmethylsulfonyl fluoride and $1 \mathrm{mM}$ EDTA) for $45 \mathrm{~min}$ at $4^{\circ} \mathrm{C}$. Approximately $30 \mu \mathrm{g}$ of total protein was loaded into each lane of 10 and $15 \%$ polyacrylamide gels and subjected to sodium dodecyl sulfate polyacrylamide gel electrophoresis. The proteins were then transferred onto nitrocellulose membranes (Novex; Invitrogen Life Technologies, Carlsbad, CA, USA) and the blots were blocked in $5 \%(\mathrm{w} / \mathrm{v})$ non-fat milk for $1 \mathrm{~h}$ at room temperature. The blots were incubated with the appropriate primary antibodies overnight at $4^{\circ} \mathrm{C}$ (GRIM-19 polyclonal mouse, 1:1,000 dilution; p-Stat3 monoclonal rabbit, 1:2,000; Stat 3 monoclonal rabbit, 1:2,000; and $\beta$-actin polyclonal mouse, 1:3,000). The blots were washed three times for $5 \mathrm{~min}$ per wash and exposed to horseradish peroxidase-conjugated secondary antibodies (Biosynthesis Biotechnology Co., Ltd.) for $2 \mathrm{~h}$. The blots were then examined using enhanced chemiluminescence reagent (Thermo Fisher Scientific, Waltham, MA, USA) and the band intensities were measured and quantified using Quantity One software (Bio-Rad, Hercules, CA, USA).

Reverse-transcription polymerase chain reaction (RT-PCR) assays. Total RNA was isolated from HeLa cells using TRIzol ${ }^{\circledR}$ reagent (Life Technologies, Rockville, MD, USA) following $24 \mathrm{~h}$ treatment with resveratrol or $48 \mathrm{~h}$ after transfection with GRIM-19 or a GRIM-19 short interfering RNA (siRNA). cDNA was generated from $1 \mu \mathrm{g}$ of total RNA using a cDNA synthesis kit (Takara Biotechnology Co., Ltd., Dalian, Lianning, China)
Table I. Oligonucleotide primer sequences used in the present study.

\begin{tabular}{|c|c|c|}
\hline Name & Sequence $\left(5^{\prime} \rightarrow 3^{\prime}\right)$ & $\begin{array}{l}\text { Amplicon } \\
\text { (bp) }\end{array}$ \\
\hline Cyclin B1 & $\begin{array}{l}\text { F: GCAGCACCTGGCTAAGAATGT } \\
\text { R: GCCTTGGCTAAATCTTGAACT }\end{array}$ & 147 \\
\hline Cyclin D1 & $\begin{array}{l}\text { F: GCGAGGAACAGAAGTGCG } \\
\text { R: AGGCGGTAGTAGGACAGGAA }\end{array}$ & 484 \\
\hline $\mathrm{Bcl}-2$ & $\begin{array}{l}\text { F: AGGATTGTGGCCTTCTTTGA } \\
\text { R: CCTACCCAGCCTCCGTTAT }\end{array}$ & 155 \\
\hline VEGF & $\begin{array}{l}\text { F: ACGGACAGACAGACAGACACC } \\
\text { R: CCCAGAAGTTGGACGAAAAGT }\end{array}$ & 176 \\
\hline$\beta$-actin & $\begin{array}{l}\text { F: AGCCTCGCCTTTGCCGATCC } \\
\text { R: ACATGCCGGAGCCGTTGTCG }\end{array}$ & 100 \\
\hline Stat3 & $\begin{array}{l}\text { F: AGTCAGTGACCAGGCAGAAGA } \\
\text { R: ATTTGTTGACGGGTCTGAAGT }\end{array}$ & 265 \\
\hline GRIM-19 & $\begin{array}{l}\text { F: CGGGACCGGAAGTGTGGGATAC } \\
\text { R: GCAGAGCATTTATTCCGTCCCAG }\end{array}$ & G 435 \\
\hline
\end{tabular}

F, forward; R, reverse. VEGF, vascular endothelial growth factor.

according to the manufacturer's instructions. Primer sequences (GeneCore, Shanghai, China) specific for cyclin B1, cyclin D1, B-cell lymphoma 2 (Bcl-2), vascular endothelial growth factor (VEGF), Stat3 and GRIM-19 were used (Table I). $\beta$-actin was used for normalization of the cDNA input levels. Following cDNA synthesis, the PCR thermal cycling profile comprised 32 cycles of denaturation $\left(95^{\circ} \mathrm{C}\right.$ for $\left.30 \mathrm{sec}\right)$, annealing $\left(56^{\circ} \mathrm{C}\right.$ for $30 \mathrm{sec})$ and extension $\left(72^{\circ} \mathrm{C}\right.$ for $\left.30 \mathrm{sec}\right)$. The reaction was terminated with a final extension step $\left(72^{\circ} \mathrm{C}\right.$ for $\left.5 \mathrm{~min}\right)$ following 32 cycles. The amplicons were separated by electrophoresis on $2 \%(\mathrm{w} / \mathrm{v})$ agarose gel and visualized by staining with ethidium bromide. Three biological replicates were analyzed for each sample point and at least three reactions were used to calculate the expression levels. Relative expression was quantified using densitometry and the Gel Image Version 3.74 System (Tianon, Shanghai, China).

Transient RNA interference and transfections. GRIM-19 was knocked down using small siRNAs, with a non-targeting siRNA used in parallel as a negative control (GenePharma Co., Shanghai, China). Primary cultures were transfected with a GRIM-19 siRNA or an irrelevant siRNA (as a control) using the X-tremeGENE HP DNA transfection reagent (Roche Diagnostics GmbH, Mannheim, Germany). After 2 days, the protein expression levels of GRIM-19, p-Stat3, Stat3 and $\beta$-actin in HeLa cells were analyzed.

Plasmid construction and DNA transfection. The human GRIM-19 sequence was amplified from HeLa cells using RT-PCR and cloned between the NotI and EcoRV sites of the Pflag-CMV ${ }^{\mathrm{TM}}-4$ mammalian expression vector. The pFLAG tag was added to the N-terminus of the GRIM-19 sequences in all the constructs. The transfection of plasmids into the cells was conducted using X-tremeGENE HP DNA transfection reagent according to the manufacturer's instructions. Following 2 days, 
the protein expression of GRIM-19, p-Stat 3 , Stat 3 and $\beta$-actin in HeLa cells was analyzed.

Cell viability assay. Cell viability was determined using MTT assays according to the manufacturer's instructions. HeLa cells were seeded in 96-well culture plates at an optimal density of $1 \times 10^{4}$ cells/well. Briefly, phosphate-buffered saline containing MTT at a final concentration of $0.5 \mathrm{mg} / \mathrm{ml}$ was added to each well following treatment with resveratrol for $24 \mathrm{~h}$, and then incubated at $37^{\circ} \mathrm{C}$ for $4 \mathrm{~h}$. The medium was gently aspirated and $150 \mu \mathrm{l}$ DMSO was added to each well. The plates were agitated for $10 \mathrm{~min}$ on a shaker to dissolve the formazan product. A well containing DMSO without cells was used as a blank control and the optical density at $490 \mathrm{~nm}$ in each well was determined using a spectrophotometer (BioTek Instruments Inc., Winooski, VT, USA).

Statistical analysis. The data were expressed as the mean \pm standard deviation for three or more independent experiments. Statistical significance was estimated using one-way analysis of variance followed by the Student-Newman-Keuls test for comparison of several groups. $\mathrm{P}<0.05$ was considered to indicate a statistically significant difference.

\section{Results}

Effect of resveratrol on the proliferation and viability of $\mathrm{HeLa}$ cells. The present study examined the effects of resveratrol on the proliferation and cell viability of HeLa cells. Analyses by MTT assays demonstrated that the treatment of HeLa cells with resveratrol (10 and $100 \mu \mathrm{M}$ ) induced cell shrinkage (Fig. 1A) and decreased cell viability in a dose-dependent manner compared with the control and DMSO groups (Fig. 1B).

Effect of resveratrol on $p$-Stat 3 and GRIM-19. The present study investigated the effects of resveratrol on Stat 3 activation and GRIM-19 expression in HeLa cells. The cells were treated with resveratrol $(10$ and $100 \mu \mathrm{M})$ for $24 \mathrm{~h}$. HeLa cells treated with resveratrol significantly induced the mRNA and protein expression of GRIM-19 (Fig. 2A-E). At the same time, p-Stat3 protein expression levels were downregulated (Fig. 2F), however, Stat3 mRNA expression levels were unaltered (Fig. 2C).

Effect of GRIM-19 on p-Stat3 and Stat3-associated genes. To understand the function of GRIM-19 on the Stat 3 signaling pathway, GRIM-19 with a FLAG tag was overexpressed. The levels of p-Stat 3 were decreased by the overexpression of GRIM-19 (Fig. 3A). The relative expression level of p-Stat3 is shown as ratio to $\beta$-actin (Fig. 3B). The expression of GRIM-19 is shown in Fig. 3C. Stat3-associated genes (cyclin B1, VEGF and $\mathrm{Bcl}-2$ ) were downregulated by the overexpression of GRIM-19. However, GRIM-19 had no effect on the transcription levels of cyclin D1 (Fig. 3D).

GRIM-19 affects the function of resveratrol on the Stat 3 signaling pathway. The cells transfected with GRIM-19 siRNA clearly downregulated the cell cytotoxicity induced by resveratrol (Fig. 4F). Furthermore, the expression of GRIM-19, p-Stat3 and Stat3 was detected (Fig. 4A-C). Resveratrol downregulated p-Stat 3 expression, while transfection with GRIM-19
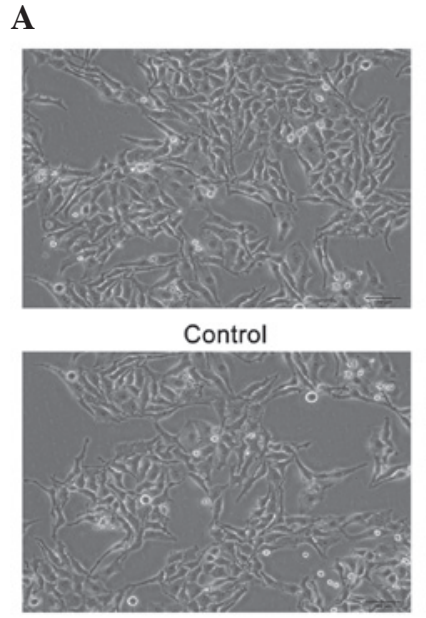

$\operatorname{RESV}(10 \mu \mathrm{M})$

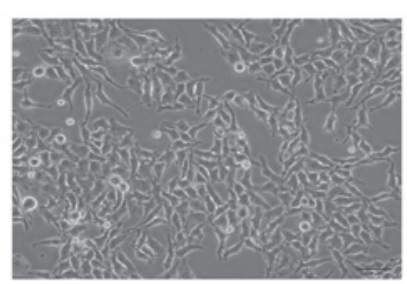

DMSO

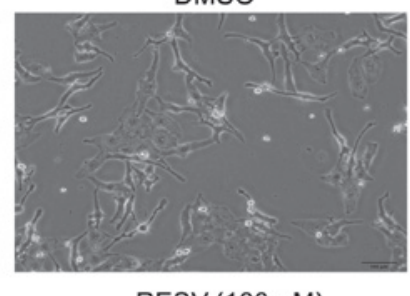

RESV $(100 \mu \mathrm{M})$

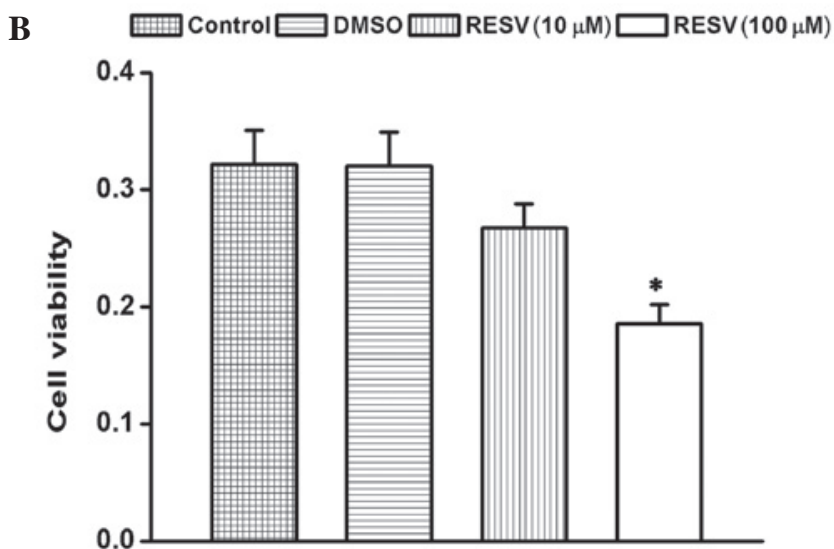

Figure 1. Effect of resveratrol on the proliferation and cytotoxicity of HeLa cells. (A) Morphological alterations in HeLa cells following treatment with resveratrol. (B) MTT assays were conducted on HeLa cells following the various treatments to determine their effects upon cell proliferation. Data are presented as the mean \pm standard deviation from three independent experiments ( $\mathrm{P}<0.05$, compared with the control group). DMSO, dimethyl sulfoxide; RESV, resveratrol.

siRNA resulted in the suppression of p-Stat3 downregulation induced by resveratrol (Fig. 4C and D). The results confirmed that GRIM-19 expression, induced by resveratrol, is involved in p-Stat3 suppression induced by resveratrol. GRIM-19 was also involved in the proliferation and cytotoxicity induced by resveratrol in HeLa cells.

\section{Discussion}

Findings of previous studies have provided new insights into the biological mechanisms of resveratrol and its associated stilbene compounds have also been investigated (20). To the best of our knowledge, the present study has demonstrated for the first time that resveratrol is able to induce the expression of GRIM-19. GRIM-19 is important in the function of resveratrol on the Stat3 signaling pathway. As previously indicated, resveratrol induced a downregulation in cell viability and induced cell-cycle arrest $(21,22)$. Furthermore, resveratrol was able to induce aberrant downstream Stat3 signaling $(23,24)$. The results of the present study have shown that resveratrol downregulated cell viability and inhibited p-Stat 3 in HeLa cells. 
A

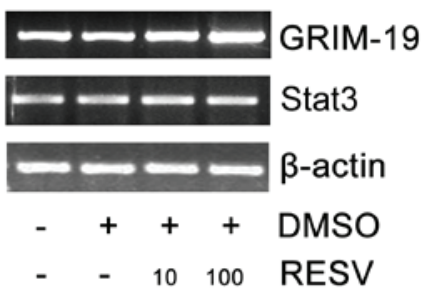

D

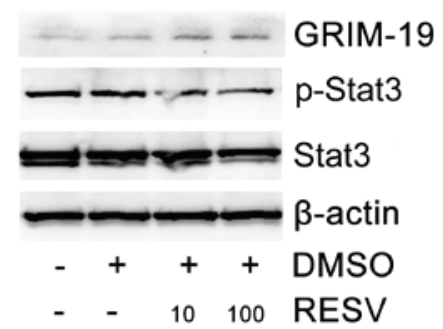

B

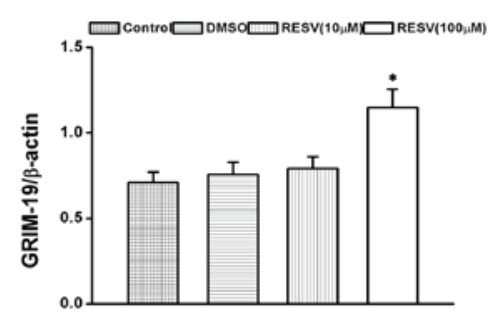

E

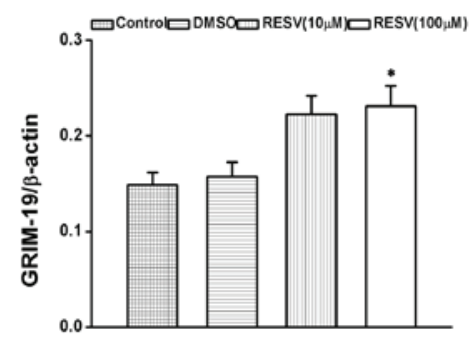

C

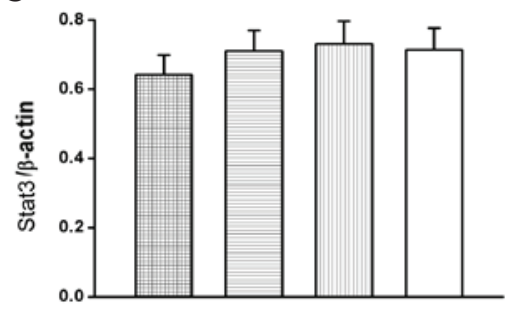

F

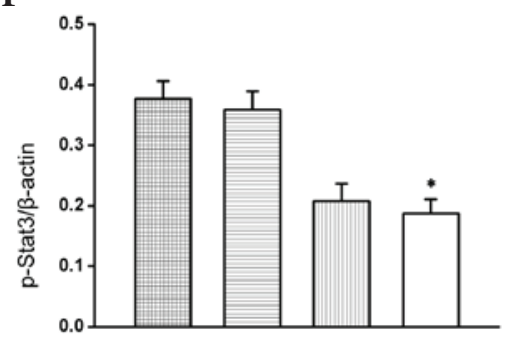

Figure 2. GRIM-19 and Stat3 mRNA and protein expression levels in HeLa cells. (A) Representative ethidium bromide-stained gels showing GRIM-19, Stat3 and $\beta$-actin amplicons. (B) Abundance of GRIM-19 mRNA is shown as a ratio to $\beta$-actin. (C) Stat 3 mRNA as a ratio to $\beta$-actin. (D) Representative western blots showing GRIM-19, p-Stat3, Stat3 and $\beta$-actin proteins. (E) Abundance of GRIM-19 protein is shown as a ratio to $\beta$-actin. (F) Abundance of p-Stat3 protein is shown as a ratio to $\beta$-actin. Data are presented as the mean \pm standard deviation from three independent samples $\left({ }^{*} \mathrm{P}<0.05\right.$, compared with the control group). GRIM-19, retinoid-IFN-induced mortality 19; Stat3, signal transducer and activator of transcription 3; DMSO, dimethyl sulfoxide; RESV, resveratrol; p-Stat3, phosphorylated Stat3.

A

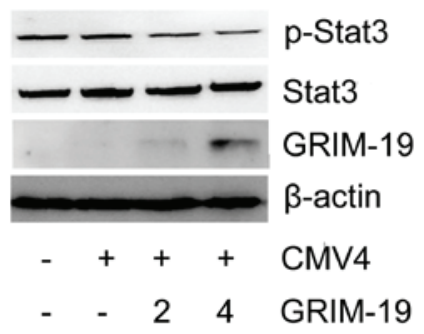

$\mathbf{D}$

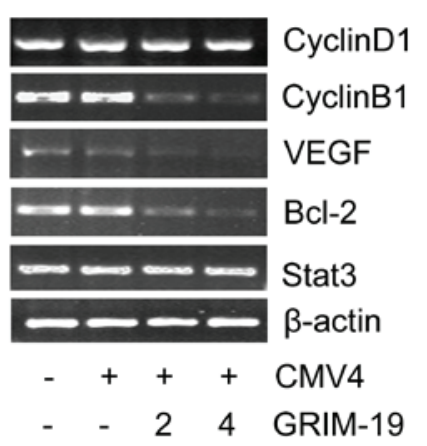

B

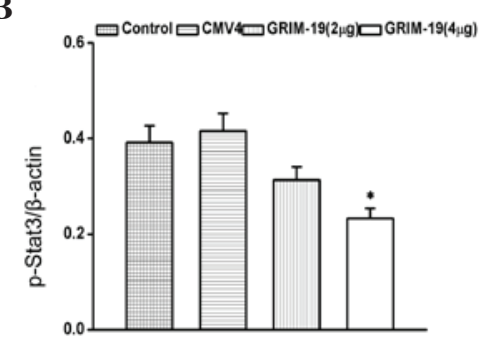

E

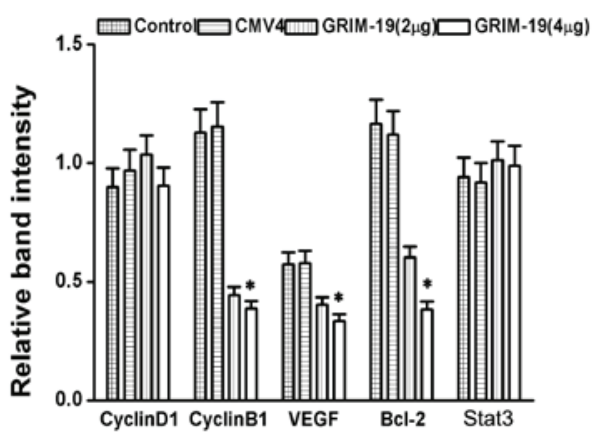

Figure 3. Effects of GRIM-19 on p-Stat3 expression. (A) Expression of p-Stat3 and GRIM-19 was detected by western blotting. The abundance of (B) p-Stat3 and (C) GRIM-19 proteins as a ratio to $\beta$-actin. (D) Representative ethidium bromide-stained gels showing Stat3, cyclin D1, cyclin B1, VEGF, Bcl-2 and $\beta$-actin amplicons. (E) Abundance of cyclin D1, cyclin B1, VEGF, Bcl-2 and Stat 3 mRNAs is shown as a ratio to $\beta$-actin. Data are expressed as the mean \pm standard deviation from three independent experiments ( ${ }^{*} \mathrm{P}<0.05$, compared with the control group). GRIM-19, retinoid-IFN-induced mortality 19 ; Stat3, signal transducer and activator of transcription 3; p-Stat3, phosphorylated Stat3; VEGF, vascular endothelial growth factor; Bcl-2, B-cell lymphoma 2; CMV4, cytomegalovirus resistance 4.

Stat3 is important in cancer development. STAT family members are phosphorylated by receptor-associated kinases in response to cytokines or growth factors. The phosphorylated STATs then translocate to the cell nucleus where they act as transcription activators and regulate the expression of target genes.

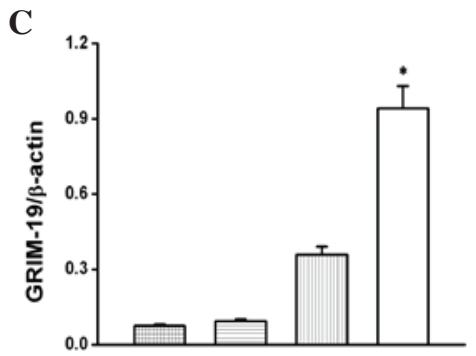

Previous studies have demonstrated that Stat 3 has an oncogenic function and that chronic Stat 3 activation is important in gastric cancer (25). Increased Stat3 activity is able to upregulate the survival signal in cancer cells (26) and specific inhibition of Stat 3 is a potentially useful therapy against various types of 
A

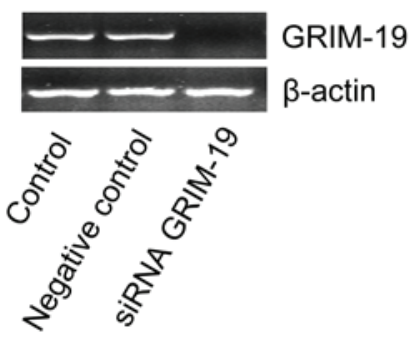

B

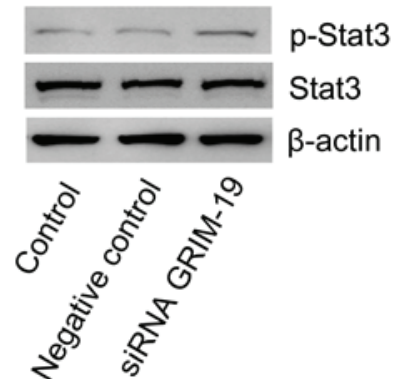

C

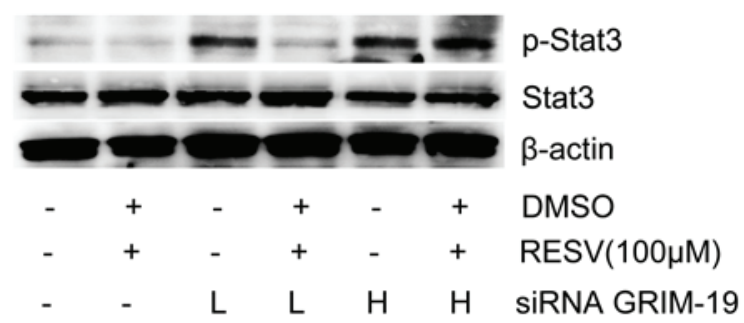

D

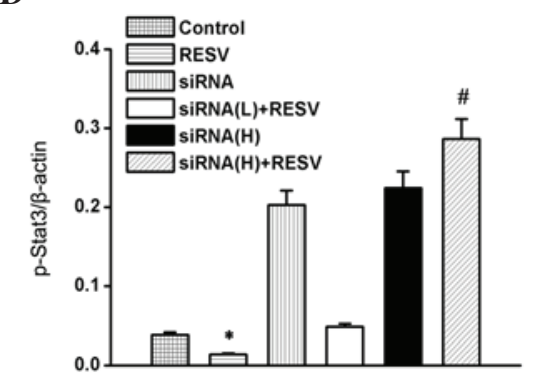

E

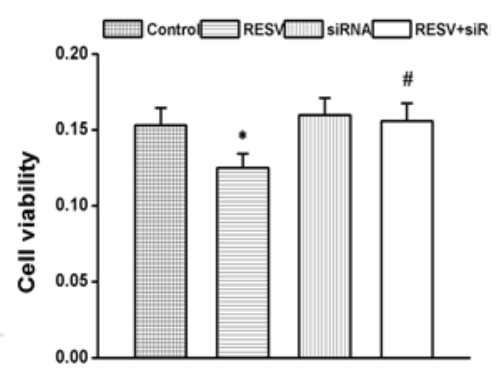

F

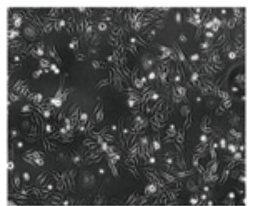

Control

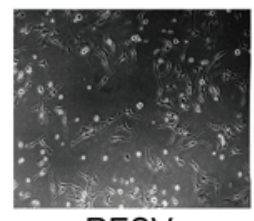

RESV

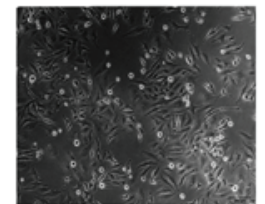

siRNA

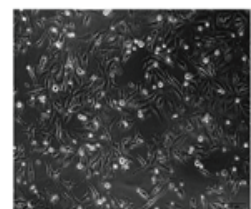

RESV+SiRNA

Figure 4. Effects of GRIM-19 siRNA on the function of resveratrol. (A) Expression of GRIM-19 and Stat3 as shown by reverse-transcription polymerase chain reaction methods and the quantity of GRIM-19 siRNAs. (B) Upregulation of the expression of the p-Stat 3 protein is shown as a ratio to $\beta$-actin. (C) Representative western blots showing p-Stat3, Stat3 and $\beta$-actin proteins in the same samples. (D) Expression of the p-Stat3 protein is shown as a ratio to $\beta$-actin. (E) HeLa cells transfected with GRIM-19 siRNA were incubated with the indicated concentrations of resveratrol for $24 \mathrm{~h}$ at $37^{\circ} \mathrm{C}$. Morphological alterations in HeLa cells following treatment with resveratrol were observed following 24 h. (F) Effects of the GRIM-19 siRNA on the function of resveratrol in HeLa cells. Cell viability was determined by the MTT bromide reduction assay. Data are presented as the mean \pm standard deviation of three independent experiments ( ${ }^{*} \mathrm{P}<0.05$, compared with the control group; ${ }^{\text {P }}<0.05$, compared with the resveratrol groups). GRIM-19, retinoid-IFN-induced mortality 19; Stat3, signal transducer and activator of transcription 3; DMSO, dimethyl sulfoxide; RESV, resveratrol; siRNA, short interfering RNA; L, low (20 $\mu \mathrm{m})$; H, high $(40 \mu \mathrm{m})$.

cancer (27). The present study found that resveratrol suppressed the expression of $\mathrm{p}$-Stat 3 and inhibited the proliferation of cancer cells. These results suggest that resveratrol suppresses HeLa cell proliferation and survival, and the anticancer function of resveratrol is partially dependent on the inhibition of Stat3 activation.

The overexpression of GRIM-19 downregulates p-Stat3 levels. At the same time GRIM-19 suppresses the transcription levels of cyclin B1, VEGF and Bcl-2. These are all downstream genes associated with cell proliferation and survival $(13,14)$. Although the function of GRIM-19 in several types of cancer and the Stat 3 signaling pathway have been previously reported $(15,19)$, the effect of GRIM-19 on the function of resveratrol and its association with the Stat 3 signaling pathway remains to be elucidated. The results from the present study clearly demonstrate that resveratrol induced the expression of GRIM-19 and suppressed the expression of p-Stat3. The GRIM-19 siRNA inhibited the suppressive effects of resveratrol on the Stat3 signaling pathway, while upregulating cell survival compared with the resveratrol group. The association between resveratrol and the Stat 3 signaling pathway remains to be elucidated. However, there is a clear correlation between the role of Stat 3 in cancer development $(8,9)$ and the function of resveratrol $(28,29)$. To the best of our knowledge, the present study has reported for the first time that resveratrol induced the expression of GRIM-19, and that GRIM-19 is important in the effects of resveratrol on HeLa cells via the abrogation of Stat3 signaling and further investigation is required in other types of cancer. In the present study, resveratrol at a high concentration $(100 \mu \mathrm{M})$ suppressed the proliferation of HeLa cells and the Stat3 signaling pathway. Previous studies have also demonstrated that resveratrol has an effect on the src-Stat 3 signaling pathway (22) and on tumor development (4). These results, in association with the results from the present study, elucidate the importance of resveratrol and its antitumor cell activities, which are partially dependent on the concentration of resveratrol (30-33).

In conclusion, the present study has reported that GRIM-19 expression, induced by resveratrol, affects the Stat 3 signaling pathway. Modulation of this signal transduction pathway contributes to the resveratrol-induced biological effects on various types of cancer (34). The present study highlights a new mechanism through which resveratrol inhibits the Stat 3 
signaling pathway. However, further investigation is required in order to fully elucidate the anti-tumorigenic effects of resveratrol.

\section{Acknowledgements}

The present study was supported by the National Natural Science Foundation of China (nos. 81070110 to M. Wei and 81100099 to P. Xin) and the Shanghai Science and Technology Innovation Research Program (no. 11410701900 to M. Wei).

\section{References}

1. Kopp P: Resveratrol, a phytoestrogen found in red wine. A possible explanation for the conundrum of the 'French paradox'? Eur J Endocrinol 138: 619-620, 1998.

2. Jang M, Cai L, Udeani GO, et al: Cancer chemopreventive activity of resveratrol, a natural product derived from grapes. Science 275: 218-220, 1997.

3. Mgbonyebi OP, Russo J, Russo IH, et al: Antiproliferative effect of synthetic resveratrol on human breast epithelial cells. Int J Oncol 12: 865-869, 1998.

4. Elattar TM and Virji AS: The effect of red wine and its components on growth and proliferation of human oral squamous carcinoma cells. Anticancer Res 19: 5407-5414, 1999.

5. Srivastava RK, Unterman TG and Shankar S: FOXO transcription factors and VEGF neutralizing antibody enhance antiangiogenic effects of resveratrol. Mol Cell Biochem 337: 201-212, 2010.

6. Bromberg J and Darnell JE Jr: The role of STATs in transcriptional control and their impact on cellular function. Oncogene 19: 2468-2473, 2000.

7. Yu H and Jove R: The STATs of cancer - new molecular targets come of age. Nat Rev Cancer 4: 97-105, 2004.

8. Macias E, Rao D and Digiovanni J: Role of stat 3 in skin carcinogenesis: insights gained from relevant mouse models. J Skin Cancer 2013: 684050, 2013.

9. Cho KH, Jeong KJ, Shin SC, et al: STAT3 mediates TGF-beta1-induced TWIST1 expression and prostate cancer invasion. Cancer Lett 336: 167-173, 2013.

10. You W, Tang Q, Zhang C, et al: IL-26 promotes the proliferation and survival of human gastric cancer cells by regulating the balance of STAT1 and STAT3 activation. PLoS One 8: e63588, 2013.

11. Han Z, Feng J, Hong Z, et al: Silencing of the STAT3 signaling pathway reverses the inherent and induced chemoresistance of human ovarian cancer cells. Biochem Biophys Res Commun 435 188-194, 2013

12. Yu W, Xiao H, Lin J and Li C: Discovery of novel STAT3 small molecule inhibitors via in silico site-directed fragment-based drug design. J Med Chem 56: 4402-4412, 2013.

13. Okamoto T, Inozume T, Mitsui H, et al: Overexpression of GRIM-19 in cancer cells suppresses STAT3-mediated signal transduction and cancer growth. Mol Cancer Ther 9: 2333-2343, 2010.

14. Nallar SC, Kalakonda S, Lindner DJ, et al: Tumor-derived mutations in the gene associated with retinoid interferon-induced mortality (GRIM-19) disrupt its anti-signal transducer and activator of transcription 3 (STAT3) activity and promote oncogenesis. J Biol Chem 288: 7930-7941, 2013.

15. Bu X, Zhao C, Wang W and Zhang N: GRIM-19 inhibits the STAT3 signaling pathway and sensitizes gastric cancer cells to radiation. Gene 512: 198-205, 2013.
16. Lufei C, Ma J, Huang G, et al: GRIM-19, a death-regulatory gene product, suppresses Stat3 activity via functional interaction. Embo J 22: 1325-1335, 2003.

17. Zhou T, Chao L, Rong G, et al: Down-regulation of GRIM-19 is associated with STAT3 overexpression in breast carcinomas. Hum Pathol 44: 1773-1779, 2013.

18. Nallar SC, Kalakonda S, Sun P, et al: Identification of a structural motif in the tumor-suppressive protein GRIM-19 required for its antitumor activity. Am J Pathol 177: 896-907, 2010.

19. Zhang Y, Hao H, Zhao S, et al: Downregulation of GRIM-19 promotes growth and migration of human glioma cells. Cancer Sci 102: 1991-1999, 2011.

20. Robertson CN, Roberson KM, Padilla GM, et al: Induction of apoptosis by diethylstilbestrol in hormone-insensitive prostate cancer cells. J Natl Cancer Inst 88: 908-917, 1996.

21. Wen S, Li H, Wu ML, et al: Inhibition of NF-kappaB signaling commits resveratrol-treated medulloblastoma cells to apoptosis without neuronal differentiation. J Neurooncol 104: 169-177, 2011.

22. Kotha A, Sekharam M, Cilenti L, et al: Resveratrol inhibits Src and Stat 3 signaling and induces the apoptosis of malignant cells containing activated Stat3 protein. Mol Cancer Ther 5: 621-629, 2006.

23. Capiralla H, Vingtdeux V, Zhao H, et al: Resveratrol mitigates lipopolysaccharide- and Abeta-mediated microglial inflammation by inhibiting the TLR4/NF-kappaB/STAT signaling cascade. J Neurochem 120: 461-472, 2012.

24. Scuto A, Kirschbaum M, Buettner R, et al: SIRT1 activation enhances HDAC inhibition-mediated upregulation of GADD45G by repressing the binding of NF-kappaB/STAT3 complex to its promoter in malignant lymphoid cells. Cell Death Dis 4: e635, 2013.

25. Giraud AS, Menheniott TR and Judd LM: Targeting STAT3 in gastric cancer. Expert Opin Ther Targets 16: 889-901, 2012.

26. Deng J, Liu Y, Lee H, et al: S1PR1-STAT3 signaling is crucial for myeloid cell colonization at future metastatic sites. Cancer Cell 21: 642-654, 2012.

27. Tkach M, Coria L, Rosemblit C, et al: Targeting Stat3 induces senescence in tumor cells and elicits prophylactic and therapeutic immune responses against breast cancer growth mediated by NK cells and CD4 ${ }^{+} \mathrm{T}$ cells. J Immunol 189: 1162-1172, 2012.

28. Sheth S, Jajoo S, Kaur T, et al: Resveratrol reduces prostate cancer growth and metastasis by inhibiting the Akt/MicroRNA-21 pathway. PLoS One 7: e51655, 2012.

29. Wang $\mathrm{H}$, Zhang $\mathrm{H}$, Tang L, et al: Resveratrol inhibits TGF-beta1-induced epithelial-to-mesenchymal transition and suppresses lung cancer invasion and metastasis. Toxicology 303: 139-146, 2013.

30. Aziz MH, Kumar R and Ahmad N: Cancer chemoprevention by resveratrol: In vitro and in vivo studies and the underlying mechanisms (Review). Int J Oncol 23: 17-28, 2003.

31. Jeong WS, Kim IW, Hu R and Kong AN: Modulation of AP-1 by natural chemopreventive compounds in human colon HT-29 cancer cell line. Pharm Res 21: 649-660, 2004.

32. Wu Y and Liu F: Targeting mTOR: Evaluating the therapeutic potential of resveratrol for cancer treatment. Anticancer Agents Med Chem 13: 1032-1038, 2013.

33. Chung MY, Lim TG and Lee KW: Molecular mechanisms of chemopreventive phytochemicals against gastroenterological cancer development. World J Gastroenterol 19: 984-993, 2013.

34. Kong AN, Yu R, Hebbar V, et al: Signal transduction events elicited by cancer prevention compounds. Mutat Res 480-481: 231-241, 2001. 